QUARTERLY OF APPLIED MATHEMATICS

VOLUME LXV, NUMBER 2

JUNE 2007, PAGES 339-355

$\mathrm{S} 0033-569 \mathrm{X}(07) 01034-1$

Article electronically published on January 16, 2007

\title{
ESTIMATES FOR THE ELECTRIC FIELD IN THE PRESENCE OF ADJACENT PERFECTLY CONDUCTING SPHERES
}

\author{
BY \\ HABIB AMMARI (Centre de Mathématiques Appliquées, CNRS UMR 7641 and Ecole \\ Polytechnique, 91128 Palaiseau Cedex, France), \\ GEORGE DASSIOS (Department of Applied Mathematics and Theoretical Physics, University of \\ Cambridge, United Kingdom), \\ HYEONBAE KANG (Department of Mathematical Sciences and RIM, Seoul National University, \\ Seoul 151-747, Korea), \\ AND \\ MIKYOUNG LIM (Centre de Mathématiques Appliquées, CNRS UMR 7641 and Ecole \\ Polytechnique, 91128 Palaiseau Cedex, France)
}

Abstract. In this paper we prove that, unlike the two-dimensional case, the electric field in the presence of closely adjacent spherical perfect conductors does not blow up even though the separation distance between the conducting inclusions approaches zero.

1. Introduction. Frequently in two phase composites, inclusions are located very closely and may even touch; see [3. It is therefore natural and important to find out if the electric field in the presence of closely spaced inclusions can be arbitrarily large or not. The purpose of this paper is to deal with the problem in three dimensions and show that, unlike the two dimensional case, the electric field is bounded regardless of the distance between the two inclusions.

In the conductivity model, the electric field is given by $\nabla u$, where $u$ is the solution to

$$
\begin{cases}\nabla \cdot\left(\chi\left(\mathbb{R}^{d} \backslash \overline{B_{1} \cup B_{2}}\right)+k_{1} \chi\left(B_{1}\right)+k_{2} \chi\left(B_{2}\right)\right) \nabla u=0 & \text { in } \mathbb{R}^{d}(d=2,3), \\ u(x)-H(x)=O\left(|x|^{1-d}\right) & \text { as }|x| \rightarrow+\infty .\end{cases}
$$

Here $H$ is a given harmonic function in $\mathbb{R}^{d}$ such that $H(0)=0, B_{1}$ and $B_{2}$ represent the inclusions, $k_{1}$ and $k_{2}$ are their conductivities, and $\chi(E)$ denotes the indicator function

Received April 30, 2006.

2000 Mathematics Subject Classification. Primary 35J25; Secondary $73 \mathrm{C} 40$.

Key words and phrases. Electric field, gradient estimates, composite materials.

E-mail address: ammari@cmapx.polytechnique.fr

E-mail address: G.Dassios@damtp.cam.ac.uk

E-mail address: hkang@math.snu.ac.kr

E-mail address: mklim@cmapx.polytechnique.fr 
of the set $E$. So the question is whether $\|\nabla(u-H)\|_{L^{\infty}}$ can be arbitrarily large as $\epsilon:=\operatorname{dist}\left(B_{1}, B_{2}\right) \rightarrow 0$.

If $B_{1}$ and $B_{2}$ are two dimensional disks and $k_{1}$ and $k_{2}$ stay away from 0 or $+\infty$, then it is proved by Bonnetier and Vogelius [4 that $|\nabla(u-H)|$ stays bounded no matter how small $\epsilon$ is. Li and Vogelius [10] extended this result and proved that the electric field stays bounded in most general setting-arbitrary number of inclusions of arbitrary shapes and in two or three dimensions, as long as the conductivities stay away from 0 and $+\infty$.

If the conductivity tends to $+\infty$ or 0 , then the situation is completely different. If the inclusions are perfect conductors $(k=+\infty)$ or insulators $(k=0)$, then the gradient blows up at the rate of $\epsilon^{-1 / 2}$, as shown by Babuška et al. [3] by numerical evidence. See also [5, 8, 11]. Recently Ammari et al. 1, 2] considered the case of two circular inclusions and rigourously derived precise estimates on the gradient clarifying the dependence on the conductivity, the radii, and the distance between the two inclusions. Yun 13] extended this result to cover two perfect conductors of arbitrary shapes in the two-dimensional case.

Unlike the two-dimensional case, not much is known in three dimensions when the conductivity is zero or infinity: Does the gradient blow up as the distance between the two inclusions tends to zero? If so, what is the blow-up rate? The purpose of this paper is to address this question. To our surprise, it turns out that if the inclusions are perfect conductors and of spherical shape, the gradient stays bounded regardless of the separation distance between them. More precisely, we prove the following theorem.

Theorem 1.1. Let $B_{1}$ and $B_{2}$ be two spheres with radius $R$ and centered at $\left(0,0, \pm R \pm \frac{\epsilon}{2}\right)$, respectively. Let $H$ be a harmonic function in $\mathbb{R}^{3}$ such that $H(0)=0$. Define $u$ to be the solution to

$$
\begin{cases}\Delta u=0 & \text { in } \mathbb{R}^{3} \backslash \overline{B_{1} \cup B_{2}}, \\ u=0 & \text { on } \partial B_{1} \cup \partial B_{2}, \\ u(x)-H(x)=O\left(|x|^{-1}\right) & \text { as }|x| \rightarrow+\infty .\end{cases}
$$

Then there is a constant $C$ independent of $\epsilon$ such that

$$
\|\nabla(u-H)\|_{L^{\infty}\left(\mathbb{R}^{3} \backslash \overline{B_{1} \cup B_{2}}\right)} \leq C .
$$

Although our result holds for this special case, we believe that it extends to arbitraryshaped conductors if their contact reduces to a point.

Theorem 1.1 is proved by first constructing an explicit solution to (1.2) using the bispherical coordinate system (section 2), and then carefully estimating the explicit solution (section 3).

2. Representation of solutions. In this section we derive an explicit formula for the solution to (1.2) using the bispherical coordinate system. Let $B_{1}$ and $B_{2}$ be the spheres as defined above. The bispherical system associated with two spheres $B_{1}$ and $B_{2}$ is defined as follows. Let

$$
a:=\frac{1}{2} \sqrt{4 R \epsilon+\epsilon^{2}} .
$$


Then, each point $x=\left(x_{1}, x_{2}, x_{3}\right)$ in the cartesian coordinate system corresponds to $(\xi, \theta, \varphi) \in \mathbb{R} \times[0, \pi] \times[0,2 \pi]$ in the bispherical system through the equations

$$
\begin{aligned}
& x_{1}=a \frac{\sin \theta \cos \varphi}{\cosh \xi-\cos \theta}, \\
& x_{2}=a \frac{\sin \theta \sin \varphi}{\cosh \xi-\cos \theta}, \\
& x_{3}=a \frac{\sinh \xi}{\cosh \xi-\cos \theta} .
\end{aligned}
$$

See [6] or [12] for the geometric meaning of each coordinate. One relevant feature is that the coordinate surface $\xi=$ constant represents the sphere centered at $(0,0, a / \tanh \xi)$ with radius $a /|\sinh \xi|$. In particular, $\partial B_{1}$ corresponds to the coordinate surface $\xi=\xi_{0}$ where

$$
\xi_{0}=\ln \left(1+\frac{\sqrt{4 R \epsilon+\epsilon^{2}}+\epsilon}{2 R}\right)
$$

and $\partial B_{2}$ to $\xi=-\xi_{0}$.

Another important feature of the bispherical system is that it is an orthogonal coordinate system and admits R-separation of variables for harmonic functions. In fact, since

$$
\begin{aligned}
\Delta h= & \frac{(\cosh \xi-\cos \theta)^{3}}{a^{2} \sin \theta}\left[\sin \theta \frac{\partial}{\partial \xi}\left(\frac{1}{\cosh \xi-\cos \theta} \frac{\partial h}{\partial \xi}\right)\right. \\
& \left.+\frac{\partial}{\partial \theta}\left(\frac{\sin \theta}{\cosh \xi-\cos \theta} \frac{\partial h}{\partial \theta}\right)\right]+\frac{(\cosh \xi-\cos \theta)^{2}}{a^{2} \sin ^{2} \theta} \frac{\partial^{2} h}{\partial \varphi^{2}}
\end{aligned}
$$

as one can see in [12, P.111], any harmonic function $h$ has a general R-separation

$$
\begin{aligned}
h(\xi, \theta, \varphi)= & \sqrt{\cosh \xi-\cos \theta} \sum_{n=0}^{+\infty} \sum_{m=0}^{n}\left[D_{n}^{m} e^{\left(n+\frac{1}{2}\right)|\xi|}+E_{n}^{m} e^{-\left(n+\frac{1}{2}\right)|\xi|}\right] \\
& \times P_{n}^{m}(\cos \theta)\left[F_{n}^{m} \cos (m \varphi)+G_{n}^{m} \sin (m \varphi)\right],
\end{aligned}
$$

where $P_{n}^{m}$ are Legendre associate functions and $D_{n}^{m}, E_{n}^{m}, F_{n}^{m}$, and $G_{n}^{m}$ are constants. See [6, Equation (38)].

Let us recall one more notion. The spherical radial distance $|x|$ is given by $r(\xi, \theta)$ which is defined by

$$
r(\xi, \theta)=a \sqrt{\frac{\cosh \xi+\cos \theta}{\cosh \xi-\cos \theta}} .
$$

Note that $r \rightarrow+\infty$ if and only if $(\xi, \theta) \rightarrow(0,0)$, and if this is the case

$$
|r(\xi, \theta) \sqrt{\cosh \xi-\cos \theta}| \leq C
$$

for some constant $C$. On the other hand, we have

$$
r(\xi, \theta) \leq 2 a, \quad \text { for } \xi>\cosh ^{-1}(2) .
$$


We now derive an explicit form of the solution to (1.2). Note that when $H \equiv 1$, the solution has been derived in [6]: $u$ is given by

$$
u=\sqrt{\cosh \xi-\cos \theta} \sum_{n=0}^{+\infty} \sqrt{2}\left[e^{-\left(n+\frac{1}{2}\right)|\xi|}-\frac{e^{\left(n+\frac{1}{2}\right) \xi}+e^{-\left(n+\frac{1}{2}\right) \xi}}{e^{(2 n+1) \xi_{0}}+1}\right] P_{n}(\cos \theta)
$$

and

$$
\frac{1}{\left|\partial B_{1} \cup \partial B_{2}\right|} \int_{\partial B_{1} \cup \partial B_{2}} \frac{\partial u}{\partial \nu}=16 \pi a \Gamma\left(\xi_{0}\right) .
$$

Here $P_{n}$ are the Legendre polynomials and the function $\Gamma$ is defined by

$$
\Gamma(\xi)=\sum_{n=0}^{+\infty} \frac{e^{(2 n+1) \xi}-1}{e^{2(2 n+1) \xi_{0}}-1} .
$$

Now, for a given entire harmonic function $H$ in $\mathbb{R}^{3}$, we define two harmonic functions $H^{e}$ and $H^{o}$, one even and the other odd with respect to $x_{3}$, by

$$
\begin{aligned}
H^{e}\left(x_{1}, x_{2}, x_{3}\right) & :=\frac{H\left(x_{1}, x_{2}, x_{3}\right)+H\left(x_{1}, x_{2},-x_{3}\right)}{2}, \\
H^{o}\left(x_{1}, x_{2}, x_{3}\right) & :=\frac{H\left(x_{1}, x_{2}, x_{3}\right)-H\left(x_{1}, x_{2},-x_{3}\right)}{2} .
\end{aligned}
$$

According to (2.3), $H^{e}$ and $H^{o}$ can be represented in the general R-separable form:

$$
\begin{aligned}
& H^{e}(\xi, \theta, \varphi)=\sqrt{\cosh \xi-\cos \theta} \sum_{n=0}^{+\infty} e^{-\left(n+\frac{1}{2}\right)|\xi|} F_{n}(a, \theta, \varphi), \\
& H^{o}(\xi, \theta, \varphi)=\sqrt{\cosh \xi-\cos \theta} \sum_{n=0}^{+\infty}(\operatorname{sgn} \xi) e^{-\left(n+\frac{1}{2}\right)|\xi|} G_{n}(a, \theta, \varphi),
\end{aligned}
$$

where

$$
F_{n}(a, \theta, \varphi)=\sum_{m=0}^{n} P_{n}^{m}(\cos \theta)\left[A_{n}^{m} \cos (m \varphi)+B_{n}^{m} \sin (m \varphi)\right]
$$

and

$$
G_{n}(a, \theta, \varphi)=\sum_{m=0}^{n} P_{n}^{m}(\cos \theta)\left[F_{n}^{m} \cos (m \varphi)+G_{n}^{m} \sin (m \varphi)\right] .
$$

Here, $\operatorname{sgn} \xi$ is defined to be

$$
\operatorname{sgn} \xi= \begin{cases}1 & \text { if } \xi>0 \\ 0 & \text { if } \xi=0 \\ -1 & \text { if } \xi<0\end{cases}
$$

Note that those terms $e^{\left(n+\frac{1}{2}\right)|\xi|}$ in (2.3) do not appear in (2.6) and (2.7). This is because $H^{e}$ and $H^{e}$ are entire harmonic functions. 
Define an even function $u^{e}$ and an odd function $u^{o}$, with respect to $x_{3}$, by

$$
\begin{aligned}
& u^{e}=\sqrt{\cosh \xi-\cos \theta} \sum_{n=0}^{+\infty}\left[e^{-\left(n+\frac{1}{2}\right)|\xi|}+\Lambda_{n}^{e}(\xi)\right] F_{n}(a, \theta, \varphi) \\
& u^{o}=\sqrt{\cosh \xi-\cos \theta} \sum_{n=0}^{+\infty}\left[(\operatorname{sgn} \xi) e^{-\left(n+\frac{1}{2}\right)|\xi|}+\Lambda_{n}^{o}(\xi)\right] G_{n}(a, \theta, \varphi)
\end{aligned}
$$

where

$$
\begin{aligned}
\Lambda_{n}^{e}(\xi) & :=-e^{-\left(n+\frac{1}{2}\right) \xi} \frac{e^{(2 n+1) \xi}+1}{e^{(2 n+1) \xi_{0}}+1} \\
& =\left(e^{\left(n+\frac{1}{2}\right) \xi}+e^{-\left(n+\frac{1}{2}\right) \xi}\right) \sum_{k=1}^{+\infty}(-1)^{k} e^{-\left(n+\frac{1}{2}\right) 2 k \xi_{0}} \\
\Lambda_{n}^{o}(\xi) & :=-e^{-\left(n+\frac{1}{2}\right) \xi} \frac{e^{(2 n+1) \xi}-1}{e^{(2 n+1) \xi_{0}}-1} \\
& =-\left(e^{\left(n+\frac{1}{2}\right) \xi}-e^{-\left(n+\frac{1}{2}\right) \xi}\right) \sum_{k=1}^{+\infty} e^{-\left(n+\frac{1}{2}\right) 2 k \xi_{0}} .
\end{aligned}
$$

TheOREM 2.1. The solution $u$ of (1.2) has the following decomposition:

$$
u=u^{e}+u^{o}
$$

Proof. One can see from the definition of $\Lambda_{n}^{e}$ and $\Lambda_{n}^{o}$ that $u$ satisfies the boundary conditions on $\partial B_{j}$ in (1.2). So we are left to show the last condition in (1.2). We show that there is a constant $M$ such that

$$
\begin{aligned}
& \limsup _{|x| \rightarrow+\infty}|x|\left|\left(u^{e}-H^{e}\right)(x)\right| \leq M, \\
& \limsup _{|x| \rightarrow+\infty}|x|\left|\left(u^{o}-H^{o}\right)(x)\right| \leq M .
\end{aligned}
$$

Thanks to (2.4), it is enough to show that

$$
\limsup _{(\xi, \theta) \rightarrow(0,0)} \frac{\left|\left(u^{e}-H^{e}\right)(\xi, \theta)\right|}{\sqrt{\cosh \xi-\cos \theta}} \leq M
$$

in order to prove (2.15), and likewise for (2.16). Put for the sake of simplicity

$$
g(\xi, \theta, \varphi):=\frac{H^{e}(\xi, \theta, \varphi)}{\sqrt{\cosh \xi-\cos \theta}}=\sum_{n=0}^{+\infty} e^{-\left(n+\frac{1}{2}\right)|\xi|} F_{n}(a, \theta, \varphi)
$$


It then follows from (2.8) that

$$
\begin{aligned}
& \frac{u^{e}-H^{e}}{\sqrt{\cosh \xi-\cos \theta}} \\
= & \sum_{n=0}^{+\infty} \sum_{k=1}^{+\infty}(-1)^{k}\left[e^{\left(n+\frac{1}{2}\right) \xi}+e^{-\left(n+\frac{1}{2}\right) \xi}\right] e^{-\left(n+\frac{1}{2}\right) 2 k \xi_{0}} F_{n}(a, \theta, \varphi) \\
= & \sum_{k=1}^{+\infty}(-1)^{k} g\left(|\xi|+2 k \xi_{0}, \theta, \varphi\right)+\sum_{k=1}^{+\infty}(-1)^{k} g\left(-|\xi|+2 k \xi_{0}, \theta, \varphi\right) \\
= & : I+I I .
\end{aligned}
$$

We then easily get from the mean value theorem

$$
\begin{aligned}
I & \leq \sum_{k=1}^{+\infty}\left|g\left(|\xi|+2 k \xi_{0}, \theta, \varphi\right)-g\left(|\xi|+2(k+1) \xi_{0}, \theta, \varphi\right)\right| \\
& \leq \sum_{k=1}^{+\infty} 2 \xi_{0} \sup _{0 \leq t \leq 2 \xi_{0}}\left|\frac{\partial g}{\partial \xi}\left(|\xi|+2 k \xi_{0}+t, \theta, \varphi\right)\right| .
\end{aligned}
$$

Recall that the coordinate surface $\xi=c$ (constant) is the sphere centered at $(0,0$, $a / \tanh c$ ) with the radius $a / \sinh c$. For all $k \geq 1, c=|\xi|+2 k \xi_{o}+t$ is bigger than $\xi_{0}$, so $\left\{\xi=|\xi|+2 k \xi_{o}+t\right\}$ is contained in $B_{1}\left(=\left\{\xi=\xi_{0}\right\}\right)$. Thus we get

$$
\begin{aligned}
& \left|\frac{\partial g}{\partial \xi}\left(|\xi|+2 k \xi_{0}+t, \theta, \varphi\right)\right| \\
& =\left|\left(\frac{\partial H^{e}}{\partial \xi} \frac{1}{(\cosh \xi-\cos \theta)^{\frac{1}{2}}}-H^{e} \frac{\sinh \xi}{2(\cosh \xi-\cos \theta)^{\frac{3}{2}}}\right)\right|_{\xi=|\xi|+2 k \xi_{o}+t} \mid \\
& \leq C\left(\xi_{0}\right)\|H\|_{\mathcal{C}^{1}\left(B_{1}\right)} \frac{1}{e^{k \xi_{0}}}
\end{aligned}
$$

where $C\left(\xi_{0}\right)$ is a constant depending on $\xi_{0}$, and hence we have

$$
I \leq \sum_{k=1}^{+\infty} C\left(\xi_{0}\right)\|H\|_{\mathcal{C}^{1}\left(B_{1}\right)} \frac{1}{e^{k \xi_{0}}} \leq M\left(\xi_{0}\right) .
$$

In the exact same way, one can show that for small $\xi$,

$$
I I \leq M\left(\xi_{0}\right)
$$

where $M\left(\xi_{0}\right)$ is a constant depending on $\xi_{0}$. 
The estimate (2.16) can be proved similarly. In fact, we have

$$
\begin{aligned}
& \left|\frac{u^{o}-H^{o}}{\sqrt{\cosh \xi-\cos \theta}}\right| \\
& =\left|\sum_{n=0}^{+\infty} \sum_{k=1}^{+\infty}\left[e^{\left(n+\frac{1}{2}\right) \xi}-e^{-\left(n+\frac{1}{2}\right) \xi}\right] e^{-\left(n+\frac{1}{2}\right) 2 k \xi_{0}} G_{n}(a, \theta, \varphi)\right| \\
& \leq \sum_{k=1}^{+\infty}\left|\frac{H^{o}\left(|\xi|+2 k \xi_{0}, \theta, \varphi\right)}{\sqrt{\cosh \left(|\xi|+2 k \xi_{0}\right)-\cos \theta}}-\frac{H^{o}\left(-|\xi|+2 k \xi_{0}, \theta, \varphi\right)}{\sqrt{\cosh \left(-|\xi|+2 k \xi_{0}\right)-\cos \theta}}\right| \\
& \leq \sum_{k=1}^{+\infty} 2|\xi| \sup _{-|\xi| \leq t \leq|\xi|}\left|\frac{\partial}{\partial \xi}\left(\frac{H^{o}}{\sqrt{\cosh \xi-\cos \theta}}\right)\right|_{\xi=2 k \xi_{0}+t} \mid .
\end{aligned}
$$

We also have

$$
\sup _{-|\xi| \leq t \leq|\xi|}\left|\frac{\partial}{\partial \xi}\left(\frac{H^{o}}{\sqrt{\cosh \xi-\cos \theta}}\right)\right|_{\xi=2 k \xi_{0}+t} \mid \leq\|H\|_{\mathcal{C}^{1}\left(B_{1}\right)} \frac{1}{e^{k \xi_{0}}},
$$

which proves (2.16). This completes the proof.

3. Gradient estimates. Let us begin by observing that the sphere $\xi=c$ is the 0 -level set of the function

$$
f\left(x_{1}, x_{2}, x_{3}\right)=x_{1}^{2}+x_{2}^{2}+\left(x_{3}-a \frac{\cosh c}{\sinh c}\right)^{2}-\left(\frac{a}{\sinh c}\right)^{2},
$$

and hence the outward unit normal $\nu$ to the sphere $\xi=c$ is given by

$$
\nu_{\xi=c}=\frac{\nabla f}{|\nabla f|}=(\operatorname{sgn} c)\left(\frac{\sin \theta \cos \varphi \sinh c}{\cosh c-\cos \theta}, \frac{\sin \theta \sin \varphi \sinh c}{\cosh c-\cos \theta}, \frac{-1+\cosh c \cos \theta}{\cosh c-\cos \theta}\right) .
$$

Since

$$
\frac{\partial\left(x_{1}, x_{2}, x_{3}\right)}{\partial \xi}=-(\operatorname{sgn} c)\left(\frac{a}{\cosh c-\cos \theta}\right) \nu_{\xi=c}
$$

we have

$$
\left.\frac{\partial u}{\partial \nu}\right|_{\xi=c}=\nabla u \cdot \nu_{\xi=c}=-\left.(\operatorname{sgn} c)\left(\frac{\cosh c-\cos \theta}{a}\right) \frac{\partial u}{\partial \xi}\right|_{\xi=c} .
$$

We now prove Theorem 1.1. During the course of the proof, we will state necessary technical lemmas, leaving their proofs to the end of this section.

Proof of Theorem 1.1. To establish the boundedness of $\nabla(u-H)$ we first observe that since $(u-H)(x) \rightarrow 0$ as $|x| \rightarrow+\infty,|\nabla(u-H)|$ attains its maximum on either $\partial B_{1}$ or $\partial B_{2}$. It then suffices to estimate $|\partial(u-H) / \partial \nu|$ on the spheres $\xi= \pm \xi_{0}$ since $u=0$ on them.

By (2.14), $u-H=\left(u^{e}-H^{e}\right)+\left(u^{o}-H^{o}\right)$, and $u^{e}-H^{e}, u^{o}-H^{o}$ are even and odd in the $x_{3}$-variable. Therefore, we have

$$
\begin{aligned}
& \left.\frac{\partial\left(u^{e}-H^{e}\right)}{\partial \nu}\right|_{\xi=-\xi_{0}}=\left.\frac{\partial\left(u^{e}-H^{e}\right)}{\partial \nu}\right|_{\xi=\xi_{0}}, \\
& \left.\frac{\partial\left(u^{o}-H^{o}\right)}{\partial \nu}\right|_{\xi=-\xi_{0}}=-\left.\frac{\partial\left(u^{o}-H^{o}\right)}{\partial \nu}\right|_{\xi=\xi_{0}},
\end{aligned}
$$


which hold because of the simple relation

$$
\left.\left(\nu_{1}, \nu_{2}, \nu_{3}\right)\right|_{\xi=-\xi_{0}}=\left.\left(\nu_{1}, \nu_{2},-\nu_{3}\right)\right|_{\xi=\xi_{0}} .
$$

Thus it is enough to consider estimates on the sphere $\xi=\xi_{0}$.

The first technical lemma is the following.

LEMma 3.1. We have

$$
\begin{aligned}
\left.\frac{\partial\left(u^{e}-H^{e}\right)}{\partial \nu}\right|_{\xi=\xi_{0}}= & \left.\left(\frac{\partial H^{e}}{\partial \nu}+\frac{\sinh \xi_{0}}{a} H^{e}\right)\right|_{\xi=\xi_{0}} \\
& -2\left(\cosh \xi_{0}-\cos \theta\right)^{\frac{3}{2}} \sum_{k=0}^{+\infty}(-1)^{k} f^{e}\left((2 k+3) \xi_{0}, \theta, \varphi\right),
\end{aligned}
$$

where

$$
f^{e}(\xi, \theta, \varphi)=\frac{1}{(\cosh \xi-\cos \theta)^{\frac{3}{2}}}\left(\frac{\partial H^{e}}{\partial \nu}(\xi, \theta, \varphi)+\frac{\sinh |\xi|}{2 a} H^{e}(\xi, \theta, \varphi)\right) .
$$

We also have

$$
\begin{aligned}
\left.\frac{\partial\left(u^{o}-H^{o}\right)}{\partial \nu}\right|_{\xi=\xi_{0}}= & \left.\left(\frac{\partial H^{o}}{\partial \nu}+\frac{\sinh \xi_{0}}{a} H^{o}\right)\right|_{\xi=\xi_{0}} \\
& +2\left(\cosh \xi_{0}-\cos \theta\right)^{\frac{3}{2}} \sum_{k=0}^{+\infty} f^{o}\left((2 k+3) \xi_{0}, \theta, \varphi\right),
\end{aligned}
$$

where

$$
f^{o}(\xi, \theta, \varphi)=\frac{1}{(\cosh \xi-\cos \theta)^{\frac{3}{2}}}\left(\frac{\partial H^{o}}{\partial \nu}(\xi, \theta, \varphi)+\frac{\sinh |\xi|}{2 a} H^{o}(\xi, \theta, \varphi)\right) .
$$

Since the formula (3.2) and (3.4) are identical except the multiplication by $(-1)^{k}$ in $(3.2)$ and $(-1)^{k}$ does not play any role in what follows, we will drop the superscript $e$ and $o$ afterwards.

Let $K$ be the convex hull of $\overline{B_{1}} \cup \overline{B_{2}}$. We prove that

$$
\left|\frac{\partial(u-H)}{\partial \nu}\right|_{\xi=\xi_{0}} \mid \leq C\|H\|_{\mathcal{C}^{2}(K)} .
$$

The first part of the right-hand side of (3.2) and (3.4) is simple to handle. In fact, one can easily see from (2.1) and (2.2) that there are constants $C_{1}$ and $C_{2}$ such that

$$
C_{1} \leq \frac{\xi_{0}}{a} \leq C_{2},
$$

and hence

$$
\left|\left(\frac{\partial H}{\partial \nu}+\frac{\sinh \xi_{0}}{a} H\right)\right|_{\xi=\xi_{0}} \mid \leq C\|H\|_{\mathcal{C}^{1}(K)},
$$

for some constant $C$.

To estimate the infinite summation of (3.2) and (3.4), we consider two different cases separately: the case when $H(x)=O\left(|x|^{2}\right)$ as $|x| \rightarrow 0$ and the case when $H(x)$ is of homogeneous degree one.

Suppose that $H(x)=O\left(|x|^{2}\right)$ as $|x| \rightarrow 0$ so that

$$
|H(x)| \leq C\|H\|_{\mathcal{C}^{2}(K)}|x|^{2} \quad \text { for } x \in K,
$$


for some constant $C$. In this case, since the sphere $\{\xi=c\}$ is contained in $B_{1} \cup B_{2}$ if $|c| \geq \xi_{0}$ and $|x|=r(c, \theta)$, we have

$$
\left|\left(\frac{\partial H}{\partial \nu}+\frac{\sinh |\xi|}{2 a} H\right)\right|_{\xi=c} \mid \leq C\|H\|_{\mathcal{C}^{2}(K)}\left(r(c, \theta)+\frac{\sinh |c|}{a} r^{2}(c, \theta)\right) .
$$

It thus follows from (3.3) and (3.5) that

$$
\begin{aligned}
& \sum_{k=0}^{+\infty}\left|f\left((2 k+3) \xi_{0}, \theta, \varphi\right)\right| \\
& \leq C\|H\|_{\mathcal{C}^{2}(K)} \sum_{k=0}^{+\infty}\left[\frac{1}{(\cosh \xi-\cos \theta)^{\frac{3}{2}}}\left(r(\xi, \theta)+\frac{\sinh |\xi|}{a} r^{2}(\xi, \theta)\right)\right]_{\xi=(2 k+3) \xi_{0}} .
\end{aligned}
$$

The desired estimate (3.6) for this case immediately follows from the following lemma.

Lemma 3.2. For $\eta>0$, there is a constant $C>0$ such that for $0<\xi_{0}<\eta$ and $\theta \in[0, \pi]$,

$$
\sum_{k=0}^{+\infty}\left[\frac{1}{(\cosh \xi-\cos \theta)^{\frac{3}{2}}}\left(r(\xi, \theta)+\frac{\sinh |\xi|}{a} r^{2}(\xi, \theta)\right)\right]_{\xi=(2 k+3) \xi_{0}} \leq \frac{C}{\left(\cosh \xi_{0}-\cos \theta\right)^{\frac{3}{2}}} .
$$

We now assume that $H$ is homogeneous of degree one. Because of the symmetry of the configuration, $x_{1}$ and $x_{2}$ play the same roles, and hence it suffices to consider the cases when $H=x_{1}$ and $H=x_{3}$.

If $H(x)=x_{3}$, we have for each $c>0$

$$
\left.\left(\frac{\partial H}{\partial \nu}+\frac{\sinh |\xi|}{2 a} H\right)\right|_{\xi=c}=\frac{-1+\cosh c \cos \theta}{\cosh c-\cos \theta}+\frac{\sinh ^{2} c}{2(\cosh c-\cos \theta)} .
$$

Thus the following lemma, together with (3.4), yields (3.6) when $H(x)=x_{3}$.

LEMMA 3.3. Let

$$
f(t):=\frac{1}{(\cosh t-\cos \theta)^{\frac{3}{2}}}\left[\frac{-1+\cosh t \cos \theta}{\cosh t-\cos \theta}+\frac{\sinh ^{2} t}{2(\cosh t-\cos \theta)}\right] .
$$

For a given $\eta>0$, there exits a constant $C>0$ such that for $0<\xi_{0}<\eta$ and $\theta \in[0, \pi]$, the following is satisfied:

$$
\left|\sum_{k=0}^{+\infty} f\left((2 k+3) \xi_{0}\right)\right| \leq \frac{C}{\left(\cosh \xi_{0}-\cos \theta\right)^{\frac{3}{2}}}
$$

If $H(x)=x_{1}$, then

$$
\left.\left(\frac{\partial H}{\partial \nu}+\frac{\sinh |\xi|}{2 a} H\right)\right|_{\xi=c}=\frac{3}{2} \frac{\sin \theta \sinh |c|}{\cosh c-\cos \theta} \cos \varphi .
$$

Thus the following lemma together with (3.2) yields (3.6) when $H(x)=x_{1}$.

Lemma 3.4. Let

$$
h(t)=\frac{\sinh t}{(\cosh t-\cos \theta)^{\frac{5}{2}}} .
$$


For a given $\eta>0$, there is a constant $C>0$ such that for $0<\xi_{0}<\eta$ and $\theta \in[0, \pi]$, the following is satisfied:

$$
\left|\sin \theta \sum_{k=0}^{+\infty}(-1)^{k} h\left((2 k+3) \xi_{0}\right)\right| \leq \frac{C}{\left(\cosh \xi_{0}-\cos \theta\right)^{\frac{3}{2}}}
$$

This completes the proof of Theorem 1.1

We now prove those lemmas used in the proof of Theorem 1.1

Proof of Lemma 3.1. Using (2.8) and (3.1), it is easy to see that

$$
\begin{aligned}
\left.\frac{\partial u^{e}}{\partial \nu}\right|_{\xi=\xi_{0}}= & \left.\frac{\partial H^{e}}{\partial \nu}\right|_{\xi=\xi_{0}}-\frac{\sinh \xi_{0}}{2 a} \sqrt{\cosh \xi_{0}-\cos \theta} \sum_{n=0}^{+\infty} \Lambda_{n}^{e}\left(\xi_{0}\right) F_{n}(a, \theta, \varphi) \\
& -\frac{\left(\cosh \xi_{0}-\cos \theta\right)^{\frac{3}{2}}}{a} \sum_{n=0}^{+\infty} \frac{\partial \Lambda_{n}^{e}}{\partial \xi}\left(\xi_{0}\right) F_{n}(a, \theta, \varphi) .
\end{aligned}
$$

Note that

$$
\begin{aligned}
\frac{\partial \Lambda_{n}^{e}}{\partial \xi}\left(\xi_{0}\right) & =\left(n+\frac{1}{2}\right) \frac{-e^{\left(n+\frac{1}{2}\right) \xi_{0}}+e^{-\left(n+\frac{1}{2}\right) \xi_{0}}}{e^{(2 n+1) \xi_{0}}+1} \\
& =-\left(n+\frac{1}{2}\right)\left[e^{-\left(n+\frac{1}{2}\right) \xi_{0}}-e^{-\left(n+\frac{1}{2}\right) \xi_{0}} \frac{2}{e^{(2 n+1) \xi_{0}}+1}\right] \\
& =-\left[\left(n+\frac{1}{2}\right) e^{-\left(n+\frac{1}{2}\right) \xi_{0}}-2 \sum_{k=0}^{+\infty}(-1)^{k}\left(n+\frac{1}{2}\right) e^{-\left(n+\frac{1}{2}\right)(2 k+3) \xi_{0}}\right]
\end{aligned}
$$

Since $\Lambda_{n}^{e}\left(\xi_{0}\right)=-e^{-\left(n+\frac{1}{2}\right) \xi_{0}}$ by (2.10), the equation (3.10) becomes

$$
\begin{aligned}
\left.\frac{\partial u^{e}}{\partial \nu}\right|_{\xi=\xi_{0}}= & \left.\left(\frac{\partial H^{e}}{\partial \nu}+\frac{\sinh \xi_{0}}{2 a} H^{e}\right)\right|_{\xi=\xi_{0}} \\
& +\frac{\left(\cosh \xi_{0}-\cos \theta\right)^{\frac{3}{2}}}{a} \sum_{n=0}^{+\infty}\left(n+\frac{1}{2}\right) e^{-\left(n+\frac{1}{2}\right) \xi_{0}} F_{n}(a, \theta, \varphi) \\
& -2 \frac{\left(\cosh \xi_{0}-\cos \theta\right)^{\frac{3}{2}}}{a} \sum_{k=0}^{+\infty}(-1)^{k} \sum_{n=0}^{+\infty}\left(n+\frac{1}{2}\right) e^{-\left(n+\frac{1}{2}\right)(2 k+3) \xi_{0}} F_{n}(a, \theta, \varphi) .
\end{aligned}
$$

Straightforward computations show that

$$
\begin{aligned}
& \sum_{n=0}^{+\infty}\left(n+\frac{1}{2}\right) e^{-\left(n+\frac{1}{2}\right) \xi_{0}} F_{n}(a, \theta, \varphi) \\
& \quad=\frac{a}{\left(\cosh \xi_{0}-\cos \theta\right)^{\frac{3}{2}}}\left[\frac{\partial H^{e}}{\partial \nu}\left(\xi_{0}, \theta, \varphi\right)+\frac{\sinh \xi_{0}}{2 a} H^{e}\left(\xi_{0}, \theta, \varphi\right)\right],
\end{aligned}
$$

and hence (3.2) follows. 
On the other hand,

$$
\begin{aligned}
\left.\frac{\partial u^{o}}{\partial \nu}\right|_{\xi=\xi_{0}} & =\left.\frac{\partial H^{o}}{\partial \nu}\right|_{\xi=\xi_{0}}-\frac{\sinh \xi_{0}}{2 a} \sqrt{\cosh \xi_{0}-\cos \theta} \sum_{n=0}^{+\infty} \Lambda_{n}^{o}\left(\xi_{0}\right) G_{n}(a, \theta, \varphi) \\
& -\frac{\left(\cosh \xi_{0}-\cos \theta\right)^{\frac{3}{2}}}{a} \sum_{n=0}^{+\infty} \frac{\partial \Lambda_{n}^{o}}{\partial \xi}\left(\xi_{0}\right) G_{n}(a, \theta, \varphi)
\end{aligned}
$$

and

$$
\begin{aligned}
\frac{\partial \Lambda_{n}^{o}}{\partial \xi}\left(\xi_{0}\right) & :=-\left(n+\frac{1}{2}\right) \frac{e^{\left(n+\frac{1}{2}\right) \xi_{0}}+e^{-\left(n+\frac{1}{2}\right) \xi_{0}}}{e^{(2 n+1) \xi_{0}}-1} \\
& =-\left(n+\frac{1}{2}\right)\left[e^{-\left(n+\frac{1}{2}\right) \xi_{0}}+2 \sum_{k=0}^{+\infty} e^{-\left(n+\frac{1}{2}\right)(2 k+3) \xi_{0}}\right] .
\end{aligned}
$$

As before, $\Lambda_{n}^{o}\left(\xi_{0}\right)=-e^{-\left(n+\frac{1}{2}\right) \xi_{0}}$, and hence (3.11) becomes

$$
\begin{aligned}
\left.\frac{\partial u^{o}}{\partial \nu}\right|_{\xi=\xi_{0}}= & \left.\left(\frac{\partial H^{o}}{\partial \nu}+\frac{\sinh \xi_{0}}{2 a} H^{o}\right)\right|_{\xi=\xi_{0}} \\
& +\frac{\left(\cosh \xi_{0}-\cos \theta\right)^{\frac{3}{2}}}{a} \sum_{n=0}^{+\infty}\left(n+\frac{1}{2}\right) e^{-\left(n+\frac{1}{2}\right) \xi_{0}} G_{n}(a, \theta, \varphi) \\
& +2 \frac{\left(\cosh \xi_{0}-\cos \theta\right)^{\frac{3}{2}}}{a} \sum_{k=0}^{+\infty} \sum_{n=0}^{+\infty}\left(n+\frac{1}{2}\right) e^{-\left(n+\frac{1}{2}\right)(2 k+3) \xi_{0}} G_{n}(a, \theta, \varphi) .
\end{aligned}
$$

Thus (3.4) follows from the identity

$$
\begin{aligned}
& \sum_{n=0}^{+\infty}\left(n+\frac{1}{2}\right) e^{-\left(n+\frac{1}{2}\right) \xi_{0}} G_{n}(a, \theta, \varphi) \\
& \quad=\frac{a}{\left(\cosh \xi_{0}-\cos \theta\right)^{\frac{3}{2}}}\left[\frac{\partial H^{o}}{\partial \nu}\left(\xi_{0}, \theta, \varphi\right)+\frac{\sinh \xi_{0}}{2 a} H^{o}\left(\xi_{0}, \theta, \varphi\right)\right],
\end{aligned}
$$

which can be derived by straightforward computations. This completes the proof.

Proof of Lemma 3.2. Let

and put

$$
h_{1}(\xi, \theta):=\frac{r(\xi, \theta)}{(\cosh \xi-\cos \theta)^{\frac{3}{2}}} \quad \text { and } \quad h_{2}(\xi, \theta):=\frac{r^{2}(\xi, \theta) \sinh \xi}{a(\cosh \xi-\cos \theta)^{\frac{3}{2}}},
$$

$$
I^{i}:=\sum_{k=0}^{+\infty} h_{i}\left((2 k+3) \xi_{0}, \theta\right), \quad i=1,2
$$

Then, we have

$$
\sum_{k=0}^{+\infty}\left[\frac{1}{(\cosh \xi-\cos \theta)^{\frac{3}{2}}}\left(r(\xi, \theta)+\frac{\sinh |\xi|}{a} r^{2}(\xi, \theta)\right)\right]_{\xi=(2 k+3) \xi_{0}}=I^{1}+I^{2} .
$$

Note that if $0<\xi_{0}<\eta$ and $\theta \in[0, \pi]$, then

$$
r(\xi, \theta)=a \sqrt{\frac{\cosh \xi+\cos \theta}{\cosh \xi-\cos \theta}} \leq \begin{cases}2 a, & \text { if } \xi \geq 2+3 \xi_{0}, \\ \frac{a \cosh (2+3 \eta)}{(\cosh \xi-\cos \theta)^{\frac{1}{2}}}, & \text { if } \xi<2+3 \xi_{0} .\end{cases}
$$


Based on the above estimates, we deal with the sums for large and small $k$ separately. Let for $i=1,2$,

$$
I^{i}=\sum_{k \geq 1 / \xi_{0}}+\sum_{0 \leq k<1 / \xi_{0}}:=I_{1}^{i}+I_{2}^{i}
$$

If $k \geq 1 / \xi_{0}$, then $(2 k+3) \xi_{0} \geq 2+3 \xi_{0}$, and hence by (3.12) we get

$$
\left|h_{i}\left((2 k+3) \xi_{0}, \theta\right)\right| \leq \frac{M a}{e^{k \xi_{0}}},
$$

for some constant $M$. It then follows from (3.7) that

$$
I_{1}^{i} \leq \frac{M a}{1-e^{\xi_{0}}} \leq C, \quad i=1,2 .
$$

If $0 \leq k<1 / \xi_{0}$, then $(2 k+3) \xi_{0}<2+3 \xi_{0}$, and hence by (3.12) we get

$$
I_{2}^{i} \leq C a \sum_{0 \leq k<1 / \xi_{0}} f_{i}\left((2 k+3) \xi_{0}\right), \quad i=1,2,
$$

for some constant $C$ depending only on $\eta$, where

$$
f_{1}(t):=\frac{1}{(\cosh t-\cos \theta)^{2}}, \quad f_{2}(t):=\frac{\sinh t}{(\cosh t-\cos \theta)^{\frac{5}{2}}} .
$$

Since $f_{1}(t)$ is a decreasing in $(0,+\infty)$, we have

$$
\begin{aligned}
\sum_{0 \leq k<1 / \xi_{0}} f_{1}\left((2 k+3) \xi_{0}\right) & \leq \frac{1}{2 \xi_{0}} \int_{\xi_{0}}^{2+3 \xi_{0}} f_{1}(t) d t \\
& =\frac{1}{2 \xi_{0}} \int_{0}^{2+2 \xi_{0}} \frac{1}{\left(\cosh \left(t+\xi_{0}\right)-\cos \theta\right)^{2}} d t \\
& \leq \frac{1}{2 \xi_{0}} \int_{0}^{2+2 \xi_{0}} \frac{1}{\left(\cosh \xi_{0}-\cos \theta\right)^{2}+\left(\cosh \xi_{0}-\cos \theta\right) t^{2}} d t \\
& \leq \frac{1}{2 \xi_{0}} \frac{1}{\left(\cosh \xi_{0}-\cos \theta\right)^{\frac{3}{2}}} \int_{0}^{+\infty} \frac{1}{1+t^{2}} d t
\end{aligned}
$$

Therefore we get from (3.7)

$$
I_{2}^{1} \leq \frac{C}{\left(\cosh \xi_{0}-\cos \theta\right)^{\frac{3}{2}}} .
$$

The estimate of $I_{2}^{2}$ is a little more involved since $f_{2}$ is not monotone. However, one can easily see that $f_{2}$ increases in $\left(0, t_{m}\right)$ and decreases in $\left(t_{m},+\infty\right)$, where $t_{m}$ is the maximum point of $f_{2}$, i.e.,

$$
t_{m}=\cosh ^{-1}\left(\frac{-\cos \theta+\sqrt{\cos ^{2} \theta+15}}{3}\right) .
$$


If $t_{m}<3 \xi_{0}$, then $(2 k+3) \xi_{0} \in\left(t_{m},+\infty\right)$ where $f_{2}$ is decreasing for all $k \geq 0$. Thus we have as before

$$
\begin{aligned}
\sum_{0 \leq k<1 / \xi_{0}} f_{2}\left((2 k+3) \xi_{0}\right) & \leq \frac{1}{2 \xi_{0}} \int_{\xi_{0}}^{2+3 \xi_{0}} f_{2}(t) d t \\
& =\frac{1}{2 \xi_{0}} \int_{\xi_{0}}^{2+3 \xi_{0}} \frac{\sinh t}{(\cosh t-\cos \theta)^{\frac{5}{2}}} d t \\
& \leq C \frac{1}{\xi_{0}} \frac{1}{\left(\cosh \xi_{0}-\cos \theta\right)^{\frac{3}{2}}} .
\end{aligned}
$$

If $t_{m} \geq 3 \xi_{0}$, the summation can be broken down into two parts according to the increase or the decrease in $f_{2}$, i.e.,

$$
\sum_{0 \leq k<1 / \xi_{0}} f_{2}\left((2 k+3) \xi_{0}\right)=\sum_{3 \leq(2 k+3)<\frac{t_{m}}{\xi_{0}}}+\sum_{\frac{t_{m}}{\xi_{0}} \leq(2 k+3)<\frac{2}{\xi_{0}}+3} .
$$

Since $f_{2}$ is increasing in the interval relevant to the first summation, we have

$$
\sum_{3 \leq(2 k+3)<\frac{t_{m}}{\xi_{0}}} f_{2}\left((2 k+3) \xi_{0}\right) \leq \frac{1}{2 \xi_{0}} \int_{3 \xi_{0}}^{t_{m}} f_{2}(x) d x+f_{2}\left(t_{m}\right),
$$

and for the second one for which $f_{2}$ is decreasing, we have

$$
\sum_{\frac{t_{m}}{\xi_{0}} \leq(2 k+3)<\frac{2}{\xi_{0}}+3} f_{2}\left((2 k+3) \xi_{0}\right) \leq f_{2}\left(t_{m}\right)+\frac{1}{2 \xi_{0}} \int_{t_{m}}^{2+5 \xi_{0}} f_{2}(x) d x .
$$

Thus we have

$$
\sum_{0 \leq k<1 / \xi_{0}} f_{2}\left((2 k+3) \xi_{0}\right) \leq \frac{1}{2 \xi_{0}} \int_{3 \xi_{0}}^{2+5 \xi_{0}} f_{2}(t) d t+2 f_{2}\left(t_{m}\right) .
$$

It then follows from the simple-to-prove inequality

$$
(t+2)(\cosh t-1) \geq t \sinh t, \quad t \geq 0,
$$

that

$$
f_{2}\left(t_{m}\right) \leq \frac{1}{\left(\cosh t_{m}-\cos \theta\right)^{\frac{3}{2}}} \frac{t_{m}+2}{t_{m}} \leq \frac{C}{\xi_{0}\left(\cosh \xi_{0}-\cos \theta\right)^{\frac{3}{2}}} .
$$

Here we used the fact $t_{m} \geq 3 \xi_{0}$. We then get from (3.16) that

$$
\sum_{0 \leq k<1 / \xi_{0}} f_{2}\left((2 k+3) \xi_{0}\right) \leq C \frac{1}{\xi_{0}} \frac{1}{\left(\cosh \xi_{0}-\cos \theta\right)^{\frac{3}{2}}} .
$$

It now follows from (3.15) and (3.17) that

$$
I_{2}^{2} \leq \frac{C}{\left(\cosh \xi_{0}-\cos \theta\right)^{\frac{3}{2}}}
$$

The proof is now completed by (3.13), (3.14), and (3.18). 
Proof of Lemma 3.3. Since

$$
\left|\frac{-1+\cosh t \cos \theta}{\cosh t-\cos \theta}\right| \leq 1 \text { and }\left|\frac{\sinh ^{2} t}{\cosh t-\cos \theta}\right| \leq \cosh t+1
$$

we have

$$
|f(t)| \leq \frac{2 \cosh t}{(\cosh t-\cos \theta)^{\frac{3}{2}}} .
$$

Note that $F^{\prime}(t)=f(t)$ where

$$
F(t):=\frac{-\sinh t}{(\cosh t-\cos \theta)^{\frac{3}{2}}} .
$$

One can easily see that $F$ is concave in $\left(0, t_{0}\right)$ and convex in $\left(t_{0},+\infty\right)$, where

$$
t_{0}:=\cosh ^{-1}\left(-5 \cos \theta+\sqrt{21 \cos ^{2} \theta+15}\right) .
$$

We separately consider the cases of $t_{0} \leq 5 \xi_{0}$ and of $t_{0}>5 \xi_{0}$.

Suppose $t_{0} \leq 5 \xi_{0}$ and let $a=(2 k+3) \xi_{0}$ for $k \geq 2$. Since $\left(a-2 \xi_{0}, a+2 \xi_{0}\right)$ is contained in $\left(t_{0},+\infty\right)$, we get from the convexity of $F$,

$$
\frac{F\left(a+2 \xi_{0}\right)-F(a)}{2 \xi_{0}} \leq f(a) \leq \frac{F(a)-F\left(a-2 \xi_{0}\right)}{2 \xi_{0}} .
$$

Summing over all $k \geq 2$ gives us that

$$
\frac{-F\left(7 \xi_{0}\right)}{2 \xi_{0}} \leq \sum_{k=2}^{+\infty} f\left((2 k+3) \xi_{0}\right) \leq \frac{-F\left(5 \xi_{0}\right)}{2 \xi_{0}} .
$$

Since

by 3.19$)$ and

$$
\left|f\left(3 \xi_{0}\right)+f\left(5 \xi_{0}\right)\right| \leq \frac{4 \cosh 5 \xi_{0}}{\left(\cosh 3 \xi_{0}-\cos \theta\right)^{\frac{3}{2}}}
$$

$$
\left|F\left(5 \xi_{0}\right)\right|+\left|F\left(7 \xi_{0}\right)\right| \leq \frac{2 \sinh 7 \xi_{0}}{\left(\cosh \xi_{0}-\cos \theta\right)^{\frac{3}{2}}} \leq \frac{C \xi_{0}}{\left(\cosh \xi_{0}-\cos \theta\right)^{\frac{3}{2}}},
$$

we have (3.8) in the case when $t_{0} \leq 5 \xi_{0}$.

If $t_{0}>5 \xi_{0}$, define $k_{0}$ as the smallest number such that $(2 k+1) \xi_{0} \geq t_{0}$. Note that $k_{0} \geq 3$ and $\left(2 k_{0}-1\right) \xi_{0}<t_{0}$. From the concavity and the convexity of $F$, we have for $k \geq k_{0}$

$$
\frac{F\left(a+2 \xi_{0}\right)-F(a)}{2 \xi_{0}} \leq f(a) \leq \frac{F(a)-F\left(a-2 \xi_{0}\right)}{2 \xi_{0}}
$$

and for $k \leq k_{0}-3$

$$
\frac{F\left(a+2 \xi_{0}\right)-F(a)}{2 \xi_{0}} \geq f(a) \geq \frac{F(a)-F\left(a-2 \xi_{0}\right)}{2 \xi_{0}},
$$

where $a:=(2 k+3) \xi_{0}$.

We estimate $I$ by splitting the summation into three parts:

$$
\sum_{k=0}^{+\infty} f\left((2 k+3) \xi_{0}\right)=\sum_{k \geq k_{0}}+\sum_{k=k_{0}-2, k_{0}-1}+\sum_{0 \leq k \leq k_{0}-3}=: I_{1}+I_{2}+I_{3} .
$$


From (3.19) and the fact that

$$
(2 k+3) \xi_{0} \leq t_{0}+2 \xi_{0}<\cosh ^{-1}(6)+2 \xi_{0} \quad \text { for } k=k_{0}-2, k_{0}-1,
$$

we obtain that

$$
\left|f\left((2 k+3) \xi_{0}\right)\right| \leq \frac{C}{\left(\cosh \xi_{0}-\cos \theta\right)^{\frac{3}{2}}} \quad \text { for } k=k_{0}-2, k_{0}-1 .
$$

Therefore,

$$
\left|I_{2}\right| \leq \frac{C}{\left(\cosh \xi_{0}-\cos \theta\right)^{\frac{3}{2}}} .
$$

To estimate $I_{1}$ and $I_{3}$, we use (3.21) and (3.22). By summing over all $k \geq k_{0}$ and all $0 \leq k \leq k_{0}-3$ separately, we have

$$
\frac{-F\left(\left(2 k_{0}+3\right) \xi_{0}\right)}{2 \xi_{0}} \leq I_{1} \leq \frac{-F\left(\left(2 k_{0}+1\right) \xi_{0}\right)}{2 \xi_{0}}
$$

and

$$
\frac{F\left(\left(2 k_{0}-1\right) \xi_{0}\right)-F\left(3 \xi_{0}\right)}{2 \xi_{0}} \geq I_{3} \geq \frac{F\left(\left(2 k_{0}-3\right) \xi_{0}\right)-F\left(\xi_{0}\right)}{2 \xi_{0}} .
$$

Thus

$$
\begin{aligned}
\frac{F\left(\left(2 k_{0}-3\right) \xi_{0}\right)-F\left(\left(2 k_{0}+3\right) \xi_{0}\right)}{2 \xi_{0}}-\frac{F\left(\xi_{0}\right)}{2 \xi_{0}} & \leq I_{1}+I_{3} \\
& \leq \frac{F\left(\left(2 k_{0}-1\right) \xi_{0}\right)-F\left(\left(2 k_{0}+1\right) \xi_{0}\right)}{2 \xi_{0}}-\frac{F\left(3 \xi_{0}\right)}{2 \xi_{0}} .
\end{aligned}
$$

Note that for some constant $C$

$$
\begin{aligned}
\left|F\left(\left(2 k_{0}-3\right) \xi_{0}\right)-F\left(\left(2 k_{0}+3\right) \xi_{0}\right)\right| & \leq 6 \xi_{0} \sup _{\left(2 k_{0}-3\right) \xi_{0} \leq t \leq\left(2 k_{0}+3\right) \xi_{0}}|f(t)| \\
& \leq \frac{C \xi_{0}}{\left(\cosh \xi_{0}-\cos \theta\right)^{\frac{3}{2}}}
\end{aligned}
$$

and

$$
F\left(\xi_{0}\right) \leq \frac{C \xi_{0}}{\left(\cosh \xi_{0}-\cos \theta\right)^{\frac{3}{2}}}
$$

Therefore

$$
I_{1}+I_{3} \geq \frac{-M}{\left(\cosh \xi_{0}-\cos \theta\right)^{\frac{3}{2}}},
$$

for some positive constant $M$. Similarly, we can show that

$$
I_{1}+I_{3} \leq \frac{M}{\left(\cosh \xi_{0}-\cos \theta\right)^{\frac{3}{2}}},
$$

and hence

$$
\left|I_{1}+I_{3}\right| \leq \frac{M}{\left(\cosh \xi_{0}-\cos \theta\right)^{\frac{3}{2}}} .
$$

This estimate together with (3.23) yields (3.8) for the case when $t_{0}>5 \xi_{0}$. This completes the proof. 
Proof of Lemma 3.4. We first note that $h$ is increasing in $\left(0, t_{m}\right)$ and then decreasing in $\left(t_{m},+\infty\right)$ where

$$
t_{m}:=\cosh ^{-1}\left(\frac{-\cos \theta+\sqrt{\cos ^{2} \theta+15}}{3}\right) .
$$

If $t_{m} \leq 3 \xi_{0},(2 k+3) \xi_{0}$ is contained in $\left(t_{m},+\infty\right)$ for all $k \geq 1$. Thus one can immediately see that

$$
h\left(3 \xi_{0}\right)-h\left(5 \xi_{0}\right) \leq \sum_{k=0}^{+\infty}(-1)^{k} h\left((2 k+3) \xi_{0}\right) \leq h\left(3 \xi_{0}\right) .
$$

Since

$$
\left|\frac{\sin \theta \sinh \xi}{\cosh \xi-\cos \theta}\right| \leq 1 \quad \text { for all } \xi \text { and } \theta,
$$

(3.9) follows from (3.25).

If $t_{m}>3 \xi_{0}$, let $m_{0}$ be the largest number such that $\left(4 m_{0}+5\right) \xi_{0} \leq t_{m}$. Then we have

$$
\begin{aligned}
\sum_{k=0}^{+\infty}(-1)^{k} h\left((2 k+3) \xi_{0}\right) & =\sum_{0 \leq m \leq m_{0}}\left[h\left((4 m+3) \xi_{0}\right)-h\left((4 m+5) \xi_{0}\right)\right] \\
& +h\left(\left(4 m_{0}+7\right) \xi_{0}\right)-\sum_{m \geq m_{0}}\left[h\left((4 m+9) \xi_{0}\right)-h\left((4 m+11) \xi_{0}\right)\right] .
\end{aligned}
$$

Thus

$$
\begin{aligned}
& \left|\sum_{k=0}^{+\infty}(-1)^{k} h\left((2 k+3) \xi_{0}\right)\right| \\
& \leq\left|h\left(3 \xi_{0}\right)-h\left(\left(4 m_{0}+5\right) \xi_{0}\right)\right|+h\left(\left(4 m_{0}+7\right) \xi_{0}\right)+h\left(\left(4 m_{0}+9\right) \xi_{0}\right) \\
& \leq 4 h\left(t_{m}\right) \leq \frac{1}{|\sin \theta|} \frac{4}{\left(\cosh 3 \xi_{0}-\cos \theta\right)^{\frac{3}{2}}},
\end{aligned}
$$

and hence (3.9) follows. The proof is now complete.

\section{REFERENCES}

[1] H. Ammari, H. Kang, and M. Lim, Gradient estimates for solutions to the conductivity problem, Math. Ann., 332 (2005), 277-286. MR2178063(2006h:78010)

[2] H. Ammari, H. Kang, H. Lee, J. Lee, and M. Lim, Optimal bounds on the gradient of solutions to conductivity problems, preprint.

[3] I. Babuška, B. Andersson, P. Smith, and K. Levin, Damage analysis of fiber composites. I. Statistical analysis on fiber scale, Comput. Methods Appl. Mech. Engrg., 172 (1999), 27-77. MR1685902 (2000a:74115)

[4] E. Bonnetier and M. Vogelius, An elliptic regurality result for a composite medium with touching fibers of circular cross-section, SIAM J. Math. Anal., 31 (2000), 651-677. MR 1745481|(2002a:35052)

[5] B. Budiansky and G.F. Carrier, High shear stresses in stiff fiber composites, J. Appl. Mech., 51 (1984), 733-735.

[6] A. Charalambopoulos, G. Dassios, and M. Hadjinicolaou, An analytic solution for low-frequency scattering by two soft spheres, SIAM J. Appl. Math., 58 (1998), 370-386. MR 1617658 (98m:35020)

[7] J.B. Keller, Stresses in narrow regions, Trans. ASME J. Appl. Mech., 60 (1993), 1054-1056.

[8] J.B. Keller, Conductivity of a medium containing a dense array of perfectly conducting spheres or cylinders or nonconducting cylinders, J. Appl. Phys., 3 (1963), 991-993.

[9] Y.Y. Li and L. Nirenberg, Estimates for elliptic systems from composite material, Comm. Pure Appl. Math., LVI (2003), 892-925. MR1990481(2004k:35097) 
[10] Y.Y. Li and M. Vogelius, Gradient estimates for solutions to divergence form elliptic equations with discontinuous coefficients, Arch. Rational Mech. Anal., 153 (2000), 91-151. MR1770682 (2001m:35083)

[11] X. Markenscoff, Stress amplification in vanishing small geometries, Comput. Mech., 19 (1996), 7783.

[12] P. Moon and D.E. Spencer, Field Theory Handbook, 2nd Ed. Springer-Verlag, Berlin, 1988. MR947546(89i:00026)

[13] H. Yun, Estimates for electric fields blown up between closely adjacent conductors with arbitrary shape, preprint, 2006. 